\title{
Retraction Note to: A study on cognitive impairment and gray matter volume abnormalities in silent cerebral infarction patients
}

\author{
Wei Luo ${ }^{1} \cdot$ Xun Jiang ${ }^{2} \cdot$ Xiaofeng $\mathrm{Wei}^{1} \cdot$ Shanshan $\mathrm{Li}^{3} \cdot$ Mengxiong $\mathrm{Li}^{1}$
}

(C) Springer-Verlag Berlin Heidelberg 2015

Retraction Note to: Neuroradiology (2015) 57:783-789

DOI 10.1007/s00234-015-1535-3

This article has been retracted by the authors due to the fact that it was published without permission and knowledge of the key authors of the study who published the same data in a different article (Yang T, Zhang L, Xiang M, Luo W, Huang J, Li M, Xiong X, Wang H. "Cognitive impairment and gray matter volume abnormalities in silent cerebral infarction" NeuroReport 2015 26(15):890-5.)

The authors of both articles have reached an agreement to retract this article. The authors apologize to the Editors and readers as well as to all authors of the study.

The online version of the original article can be found at http://dx.doi.org/ 10.1007/s00234-015-1535-3.

Shanshan Li

lishanshanbel@163.com

1 Biomedical Engineering Laboratory, The First Affiliated Hospital of Yangtze University, Jingzhou, Hubei, People's Republic of China

2 Biomedical Engineering Laboratory, Renmin Hospital of Wuhan University, Wuhan, Hubei, People's Republic of China

3 JingZhou City Central Blood Bank, 199 Jiangin Middle, Jingzhou, Hubei, People's Republic of China 434000 